\title{
Bistable perception: neural bases and usefulness in psychological research
}

\author{
Percepción biestable: bases neurales y utilidad en la investigación psicológica \\ Guillermo Andrés Rodríguez Martínez ${ }^{1,2}$, Henry Castillo Parra ${ }^{2}$
}

\begin{abstract}
Bistable images have the possibility of being perceived in two different ways. Due to their physical characteristics, these visual stimuli allow two different perceptions, associated with top-down and bottom-up modulating processes. Based on an extensive literature review, the present article aims to gather the conceptual models and the foundations of perceptual bistability. This theoretical article compiles not only notions that are intertwined with the understanding of this perceptual phenomenon, but also the diverse classification and uses of bistable images in psychological research, along with a detailed explanation of the neural correlates that are involved in perceptual reversibility. We conclude that the use of bistable images as a paradigmatic resource in psychological research might be extensive. In addition, due to their characteristics, visual bistable stimuli have the potential to be implemented as a resource in experimental tasks that seek to understand diverse concerns linked essentially to attention, sensory, perceptual and memory processes.

Resumen

Las imágenes biestables tienen la posibilidad de ser interpretadas de dos maneras diferentes. Dadas sus características físicas, ellas admiten dos percepciones diferentes, asociadas a procesos moduladores de tipo top-down y bottom-up. A partir de una revisión narrativa exhaustiva tendiente a recabar los modelos teóricos y los fundamentos propios de la biestabilidad implicada en la observación de estas imágenes, el presente artículo teórico compila no sólo nociones que se entrecruzan en el entendimiento de este fenómeno, sino también las diversas clasificaciones y usos de este tipo de imágenes en la investigación psicológica, junto a una explicación detallada de diversos correlatos neurales implicados en la reversibilidad perceptual. Se concluye cuan extenso puede ser el uso de las imágenes biestables como recurso paradigmático, y que, por sus características, ellas tienen ricas posibilidades de ser utilizadas en tareas experimentales tendientes a desentrañar diversas inquietudes circunscritas esencialmente a procesos atencionales, sensoriales, perceptuales y de memoria.
\end{abstract}

\section{Keywords}

bistable perception; visual perception; bistable images; psychological research.

Palabras Clave

percepción biestable; percepción visual; imágenes biestables; investigación psicológica.

${ }^{1}$ Escuela de Publicidad - Universidad de Bogotá Jorge Tadeo Lozano, Bogotá, Colombia

${ }^{2}$ Facultad de Psicología - Universidad de San Buenaventura de Medellín, Colombia.

*Corresponding author: Guillermo Andrés Rodríguez Martínez. guillermo.rodriguez@utadeo.edu.co. Carrera 4 No. 22-61, Escuela de Publicidad, Universidad de Bogotá Jorge Tadeo Lozano, Bogotá, Colombia.

Manuscript received 02-02-2018; revised 22-06-2018; accepted 18-07-2018.

\section{Introduction}

It is known that the identification of regions in the brain that are associated with a conscious perception of visual stimuli is one of the aims clearly defined in neuroscience (Cumming \& Parker, 1997). It has also been stated that visual search tasks have used bistable images in order to study multiple psychological processes such as priming effect, semantic modulation, unity assumption, attention, top-down and bottom up processes, and perception, among others. The reason why these images have been taken into account in psychological research is that bistable perception involves diverse concepts of cognitive neuroscience as well as mechanisms that allow for a dissociation of processes linked to sensory stimulation from those that are connected to conscious perception (Borisyuk, Chik, \& Kazanovich, 2009; Weilnhammer, 
Ludwig, Sterzer, \& Hesselmann, 2014; Yeh, Hsiao, Chen, \& Spence, 2011). In this regard, bistable images have become a paradigmatic category that makes it possible to investigate different psychological phenomena. In fact, bistable stimuli have been extensively used to understand factors entailed in visual perception and consciousness (Leopold \& Logothetis, 1999; Pressnitzer \& Hupé, 2006; Sterzer, Kleinschmidt, \& Rees, 2009; Sterzer \& Rees, 2009).

\section{Method}

In order to carry out a detailed literature review of bistable perception, its nature, its foundation, applications and foresight, a scientific literature search was performed by using scientific databases such as SCOPUS, Science Direct, DOAJ and PubMed. The key words were "bistable perception," "multistable perception," "ambiguous figures," "perceptual reversals," "ambiguity in visual perception," "perceptual reversibility," "eye-tracking studies" and "bistable images paradigm." Studies on bistable and multistable perceptions were taken into account, as well as texts that referred to methodological and technical factors or research paradigms. All duplicates were identified and duly removed by using automatic and manual procedures (combined).

This search led to the collection of 89 references, including empirical-analytical studies (78) and review articles (11). In addition to the inclusion of texts that refer to bistable images as a paradigmatic model, articles related to experimental tasks associated with the study and understanding of the phenomenon of bistable perception were considered. The comprehensive scientific literature that was found also involves several underlying factors of multistable phenomena such as perception reversibility, modulation of visual perception, and different types of reversible figures. There were deferral criteria for all the results that were obtained. First, articles that were about bistable (or multistable) perception relating to auditory perception were discarded, leaving only articles that contained information on visual perception (in association with bistable or multistable perception). Secondly, it was estimated that the notions "perceptual reversals" and "perceptual reversibility" were necessarily linked to the perceptual bistable phenomenon. Nevertheless, every article that came from using these key words was reviewed in such a way that they were about bistable visual perception. As far as the key word "eye-tracking studies" is concerned, it was necessary to remove articles that were not about eye-movement patterns in viewing ambiguous figures, leaving just three articles. Given the fact that a preliminary search showed that there are a few scientific articles on eye-tracking studies relating to bistable visual perception, the present text draws together articles published since 1980, a date after which articles that are points of reference on eye movements in viewing bistable images were published. Thus, in order to define a search time interval, more than 30 years were taken into account, based on the publication date of the article by Gale \& Findlay, a mandatory reference in relation to eye movements during bistable perception (it is one of the two book sections that were included; these chapters are, indeed, scientific papers). 77 articles that were found (out of 89) are dated from 2002 to 2017 (see in Figure 1). The search was done from January 2016 to December 2017.

\subsection{Bistable perception: essential notions and princi- ples}

The perceptual phenomenon by which an observer perceives the same stimuli in two different ways is known as bistable perception (Borisyuk et al., 2009; Clément \& Demel, 2012; Grossmann \& Dobbins, 2006; Sterzer \& Rees, 2009; van Loon et al., 2013). Without any variations in the stimuli (in the case of a static image), or with movement or fluctuations (in the case of bistable or multistable dynamic visual stimuli), the observer's perception often alternates between two possible interpretations, because the stimuli offer several interpretation possibilities that cannot be perceived simultaneously (MorenoBote, Rinzel, \& Rubin, 2007; Schauer, Kanai, \& Brascamp, 2016; Weilnhammer, Stuke, Hesselmann, Sterzer, \& Schmack, 2017; Xiaogang et al., 2017). Given that bistable visual stimuli admit two (or more, in the case of multistable images) possible percepts (Sterzer, Russ, Preibisch, \& Kleinschmidt, 2002), they can also be called ambiguous figures (Brouwer \& van Ee, 2006; Okazaki, Kaneko, Yumoto, \& Arima, 2008). Likewise, the phenomenon of visual perceptual bistability can also be known as visual bistability (Intaitè, Koivisto, \& CasteloBranco, 2014), where a shift of the possible percepts of bistable image necessarily emerges, commonly called perceptual reversal (Fagard et al., 2008; Sandberg et al., 2014). These changes between one percept and the other are what make bistability arise. It is essentially caused by alterations in patterns while observing the bistable stimulus and is also understood as a variation in gestaltic processes of organization (Pressnitzer \& Hupé, 2006).

The concept of "bistability" applies to several processes in which alternations are possible such as bistable perception and binocular rivalry. Furthermore, there are multistable phenomena. For them, the word "multistability" is often used. In this regard, it is possible to define multistability as the phenomenal experience of alternating between perceptual representations (Castelo-Branco \& Castelhano, 2015). Thus, each bistable phenomenon can be considered multistable and the act of alternating between two different visual percepts while observing a constant ambiguous input is called bistable visual perception. To understand this phenomenon, diverse factors that allow the perceptual alternation that is typical of this type of images have been considered. On one hand, 


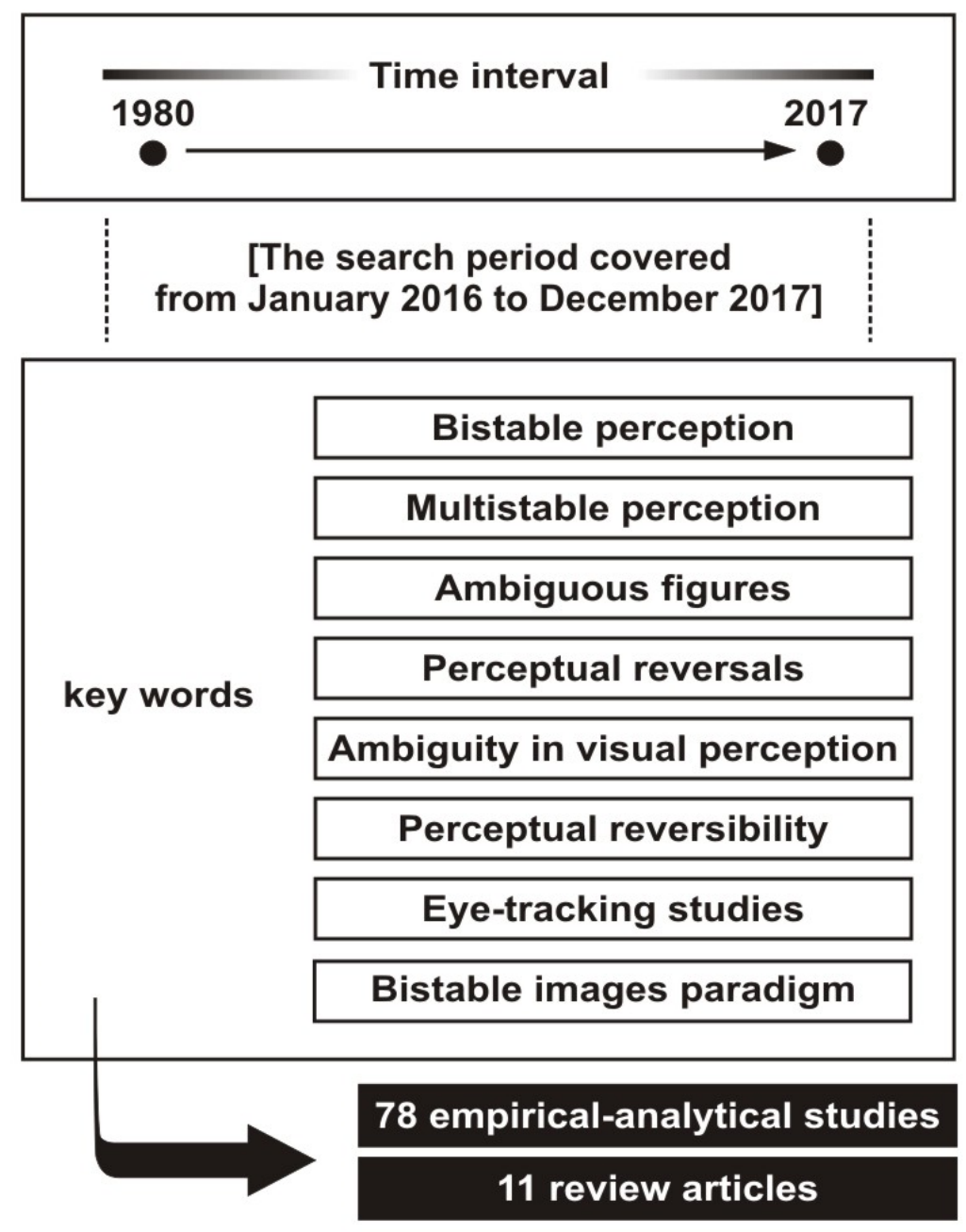

\begin{tabular}{ll|l|}
$\Rightarrow$ & $2002-2017$ & 77 articles (out of 89 ) \\
$\Rightarrow$ & $1998-2015$ & 11 review articles \\
\cline { 2 - 3 } & $1981-2017$ & 78 empirical-analytical studies \\
\hline
\end{tabular}

Figure 1. Review process diagram.

it is evident that the physical characteristics of bistable images generate ambiguity, in such a way that the resulting perceptual configuration depends not only on the way the stimuli are being observed, but also on the areas of the image in which the eyes are fixing and the observer's visual trajectory during observation (Gale \& Findlay, 1983; García-Pérez, 1989, 1992; Hsiao, Chen, Spence, \& Yeh, 2012). Thus, when the physical aspects of the stimuli are the ones that impact perception (as in shared surroundings for the two possible interpretations and defined plans, contrary and complementary shades, rotations that lead to the creation of optical illusions, among others), it is inferred that what is manifested there is a modulation of visual perception, in this case, the bottom-up type (Hsiao et al., 2012; Meng \& Tong, 2004), which is also understood as an endogenous influence (Brouwer \& van Ee, 2006). Secondly, it has been widely demonstrated that the perception of a bistable image is also conditioned by a processing of information different from the physical characteristics of the stimulus, that is from the concepts and predispositions that are integrated into the perceptual process, modulating the result of what is being seen. This sort of modulation is known as top-down type processing in terms of basic psychological processes, where the interpretation of a bistable stimulus is established by information previously stored in the memory, or by information that additionally forays in the perceptual system, generating 


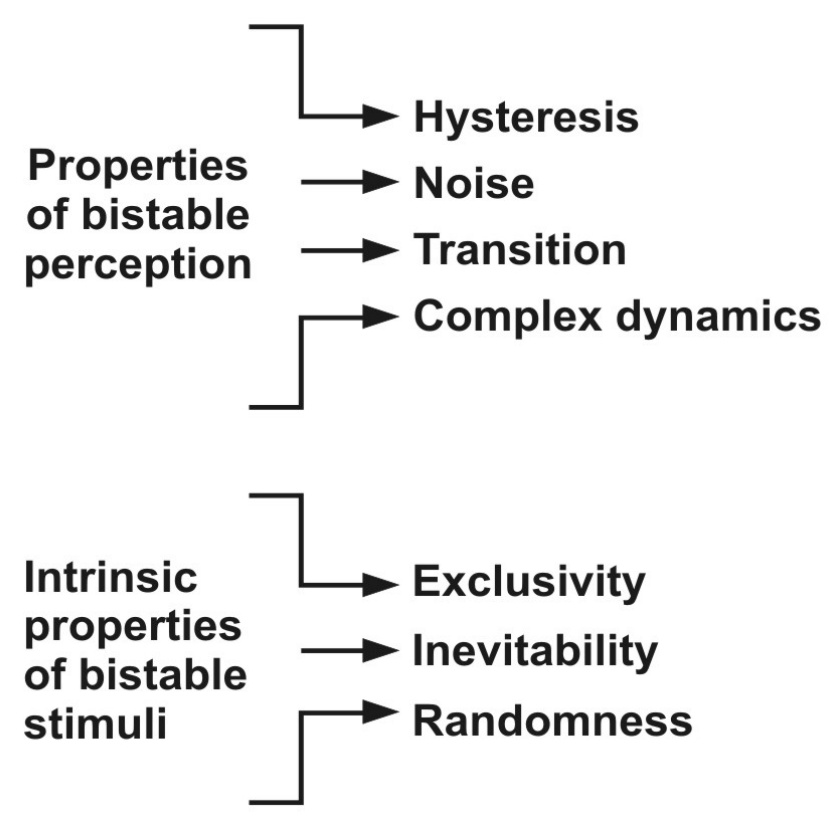

Figure 2. Properties of bistable perception and bistable stimuli.

an interpretative echo at the moment of semantically defining the percept.

The alternations between the two possible percepts are related to attention processes whereby eye movements can be responsible for the exertion of voluntary control (van Dam \& van Ee, 2006). The design of visual tasks that are based on ambiguous images must consider the fact that during prolonged viewing a sudden alternation emerges as long as the two interpretations are equally likely to occur (Kornmeier \& Bach, 2005). In this regard, the type of visual bistable image can exert an influence on the possibility of switching percepts. Furthermore, the perceptual system enables the maintenance of attention to stimulus properties with a specific expected reward. Thus, the system itself is able to prevent interferences from distracting aspects of the stimulus (Piantoni, Romeijn, Gomez-Herrero, Werf, \& Someren, 2017). On the other hand, perceptual alternation has been explained by a mutual inhibition circuit in which there is an adaptation of neurons that emerge as a consequence of neuronal fatigue (Kogo, Hermans, Stuer, van Ee, \& Wagemans, 2015). All these factors give visual tasks more or less complexity depending on the ambiguous stimuli that are going to be used.

The bottom-up and top-down approaches for understanding perceptual bistability converge in the idea that visual perception leads to a dynamic function of the brain that is modulated not only because of basic sensorial processing (bottom-up), but also because of exogenous references to the distal stimulus which impact its interpretation (top-down processing). In this regard, the occurrence of perceptual reversals poses bi-causality (Ko- rnmeier, Hein, \& Bach, 2009). Additionally, alternation between percepts can be involuntary (especially when bottom-up modulations are presented), but it can also be voluntary, not only when observers execute attention controls that direct their perception (Intaitè, Koivisto, Rukšènas, \& Revonsuo, 2010), but also when they perform eye movements (voluntarily or involuntarily), which can impact the observed percepts (van Dam \& van Ee, 2006).

It has been stated that there can be an interaction of bottom-up and top-down processes during the perception of a bistable image (Kornmeier et al., 2009). These processes seem to influence the way an observer independently perceives an ambiguous image (Intaite, Noreika, Šoliūnas, \& Falter, 2013). Considering that bottom-up factors are related to both the features of the stimulus and the way the image is being viewed (Hsiao et al., 2012), the perception of a bistable image tends to alternate stochastically (Denham et al., 2012). That switching is usually involuntary, nevertheless, there can be a control by the "will" (Borisyuk et al., 2009). The interaction between top-down and bottom-up levels of processing is linked to cycles of adaptation, inhibition, and recovery processes. Thus, perceptual reversals can be caused by feedback operations that occur from central mechanisms to lower level sensory activities, in such a way that selective attention can be directed (Intaite et al., 2013; Leopold \& Logothetis, 1999). The comparison of the models of perceptual bistability shows that they are founded in the idea of a dynamic bistability, understanding this as the presentation of a bistable visual stimulus that possesses two (or more) states inside the 
space of a dynamic model (Borisyuk \& Hoppensteadt, 2004).

Other theoretical approaches that explain perceptual alternation include the following: first, parameters of slow adaptation, where it goes from a stable state to another (hysteresis); second, a rupture of the symmetry where an irregularity given by the perceptual alternation is manifested (noise); third, transitions and metastability, which imply transitional phases that lead to perceptual bistability and perceptual jumps; fourth, probability models that describe bistable perception depending on randomized variables; and finally, complex systems (complex dynamics in a deterministic system), where reversals are irregular and subject to dynamic properties in a plan that consists of two states (Borisyuk et al., 2009).

On the other hand, intrinsic properties of bistable stimuli have been estimated (see Figure 2), namely exclusivity, inevitability and randomness. Exclusivity means that each perceived percept will be seen in a unique way; that is to say, the two possible percepts of the bistable image will never be perceived simultaneously. Inevitability accounts for a passive and automatic process through which perceptual alternations emerge. And randomness is associated with involuntary attention processes, mediated by random cerebral mechanisms where the timing for the execution of such process corresponds to the brain's own decisions, for example saccadic eyemovements (Leopold \& Logothetis, 1999).

\subsection{Types of bistable images}

The classification of bistable visual stimuli that has been carried out has taken into account diverse mechanisms of perceptual organization. In this regard, psychological process integrated to perceptual systems (like attention and the modulating mechanisms of visual perception) have contributed to the classification of bistable images. Some bistable images are not linked to establishing a different meaning for each single possible percept (e.g. Necker cube) whereas others imply disengaging from the first percept so as to assign a new meaning to the new perception (see Figure 3). Additionally, reversibility of meaning needs to direct attention to eliminate the original interpretation. In other words, reinterpreting the bistable visual stimulus can be under the control of executive processes of selective attention, inhibitory control and alternation (Bialystok \& Shapero, 2005).

The criteria used for the establishment of a classifier correspond to three fundamental notions, namely: first, the alternation between one figure and another, where the perceived one acquires the connotation of perceived figure and the other emerges as a background; second, the alternation as a sense of direction (change in the sense of perspective or change in the direction of the movement); and third, an alternation between the meaning of what is perceived where the bistable stimulus seemed to contain simultaneously two percepts with different semantic contents, but they can only be perceived individually and never simultaneously. This is how three types of bistable images can be established (Bialystok \& Shapero, 2005; Long \& Toppino, 1981): 1. In figure-ground reversal images are characterized by the fundamental fact by which one of the percepts is assumed as the background of the whole image, while the other stands out from the background and becomes the perceived figure (the vase-face illusion is an example); 2 . The images known as In perspective reversals have the particular feature that while the observer experiences each perceptual reversal, they experience a jump in the direction of the perspective of the image so that the positional sense of the images shifts, as in the case of the Necker's cube; and, 3. Bistable images In meaning-content reversals. This type of bistable images facilitates the alternation of two percepts that are presented in the same level of prominence, but where each one is different in terms of the shape and its semantic content (meaning), as can be seen in the Boring's image My girlfriend or my mother-in-law.

But aside from this classification, other classifications must also be estimated, depending on other criteria, like the number of possible percepts of the image and whether the stimulus is shown statically or in movement. Thus, regarding the number of percepts as criteria, apart from bistable images, there are tristable stimuli, a type of multistable stimuli that contains three different possible percepts (Long \& Batterman, 2012; Naber, Gruenhage, \& Einhäuser, 2010). In this sense, multistability implies assuming a particular phenomenon linked to the generation of diverse percepts which lead to the notion of multistable perception (Gori, Giora, \& Pedersini, 2008; Meso \& Masson, 2015).

Referring to the criterion that deals with the movement of the stimulus, the classifier considers two types of ambiguous images, static and dynamic. An example of the latter is "The silhouette spinner", a bistable image in movement where a woman who rotates can be perceived as rotating either clockwise and counterclockwise (Liu, Tzeng, Hung, Tseng, \& Juan, 2012). Then, the possibility of perceptual reversals emerges, where reversibility is given from the shift in direction of the movement (Jackson, Cummins, \& Brady, 2008). As another criterion for categorization, we have color, with bistable images in grayscale, black and white and color (García-Pérez, 1992). Regardless of the type of bistable image, it is assumed that the percept that is mostly perceived will be the dominant percept (Kogo et al., 2015).

\subsection{Neural bases of bistable perception}

The phenomenon of bistability in vision leads to a neural activity that implies operativity of diverse neural substrates (see Figure 4) and several integrated perceptual processes (Munhall, Ten Hove, Brammer, \& Paré, 2009). The use of invasive electrophysiological techniques has been considered the most suitable instrument to find 

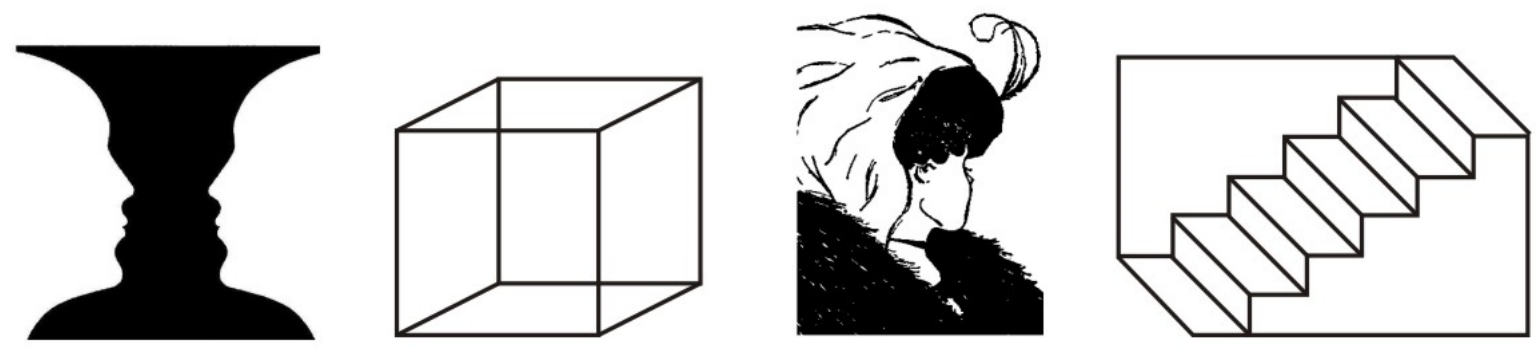

Figure 3. Types of bistable images.

From left to right: 1 . The figure The vase-face illusion - Rubin's vase (In figure-ground reversals); 2. Necker's cube (In perspective reversals); 3. My girlfriend or my mother-in-law (In meaning-content reversals); 4. The Schröder reversible staircase (In perspective reversals).

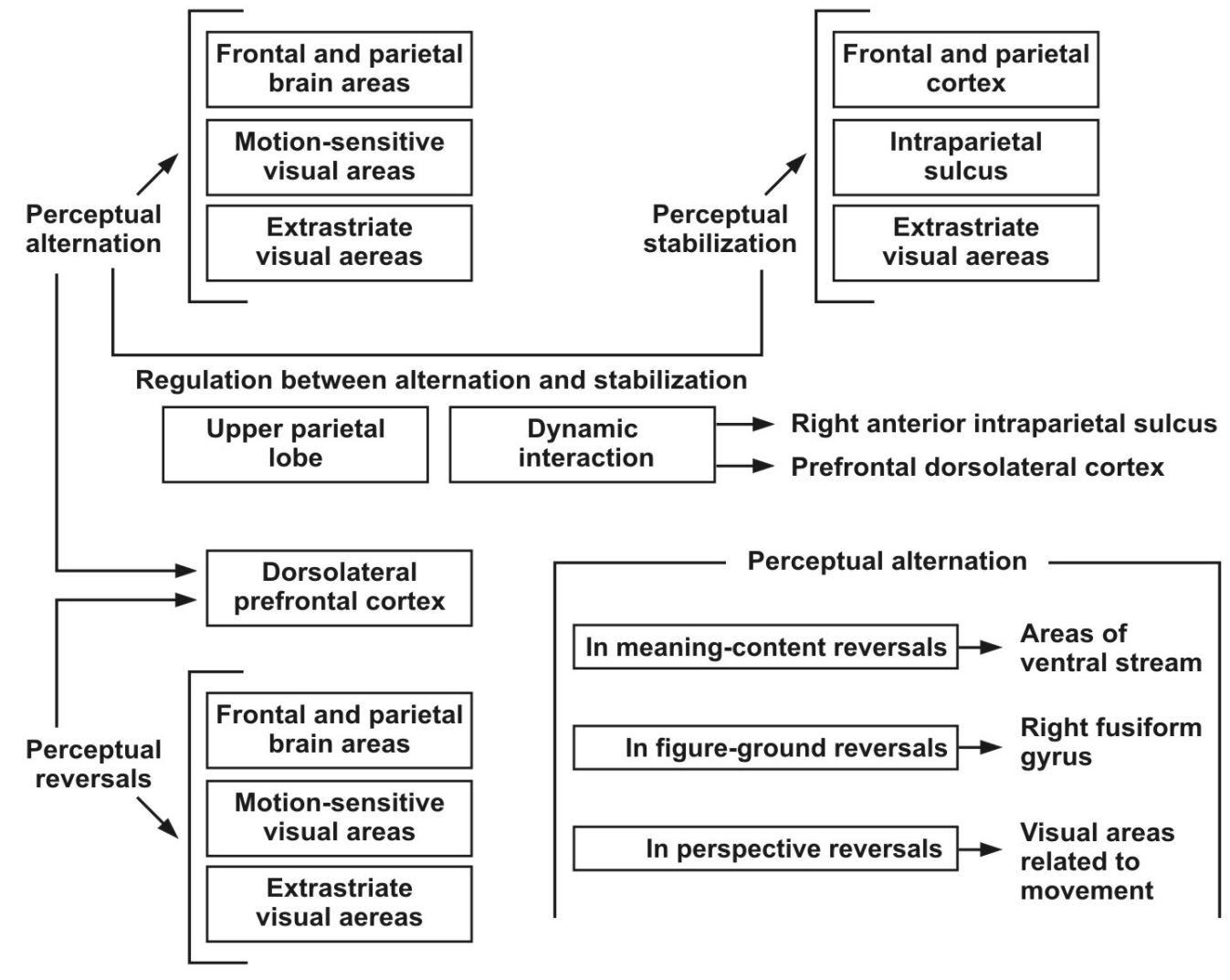

Figure 4. Neural bases of bistable perception.

explanations from the point of view of perceptual neural activity and its underlying factors. Nevertheless, and given that these techniques require the insertion of electrodes through the skull, they turn out to be dangerous methods, to the point that they can cause infections and injuries (Sterzer \& Rees, 2009). Taking that into account, functional magnetic resonance imaging - fMRI has become the most common technique to establish neural correlates with bistable perception in human subjects (Sterzer \& Rees, 2009). Besides this technique, electroencephalography (EEG), magnetoencephalography (MEG) and transcranial magnetic stimulation (TMS) are also recognized as useful and widely used. Through these methods, neural activity is measured (indirectly) in groups of neurons, which suggests extreme caution and methodic discipline to generate interpretations (Sterzer \& Rees, 2009). FMRI studies suggest that in relation to the perceptual process manifested during the observation of a bistable image, the fronto-parietal region of the brain plays a determining role in terms of solving ambiguity and defining a percept (Kleinschmidt, Büchel, Zeki, \& Frackowiak, 1998; Megumi, Bahrami, Kanai, \& Rees, 2015). Moreover, TMS studies of the parietal cortex have demonstrated causality in diverse areas in the 


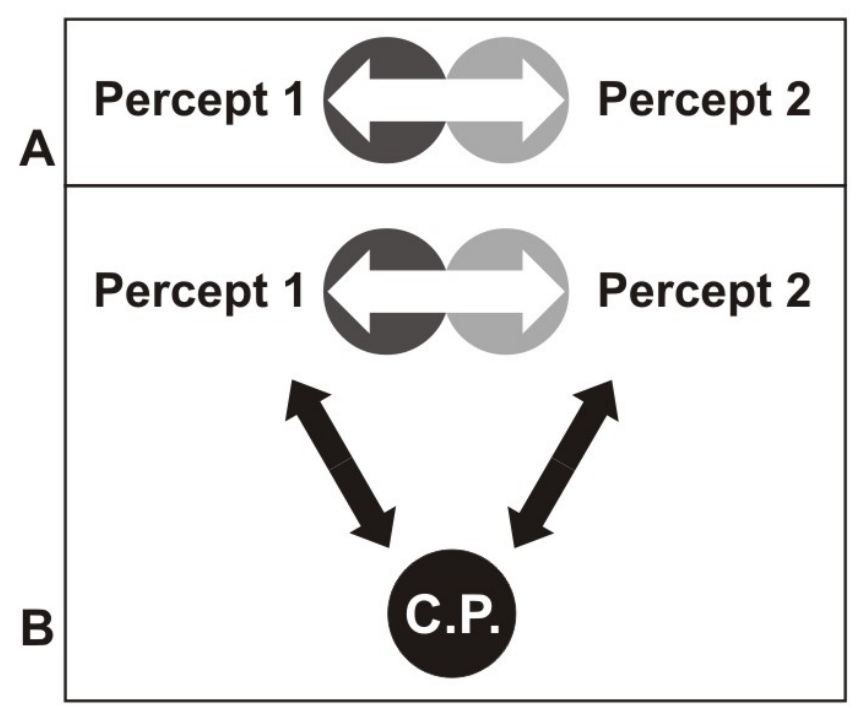

Figure 5. Low-level and High-level models.
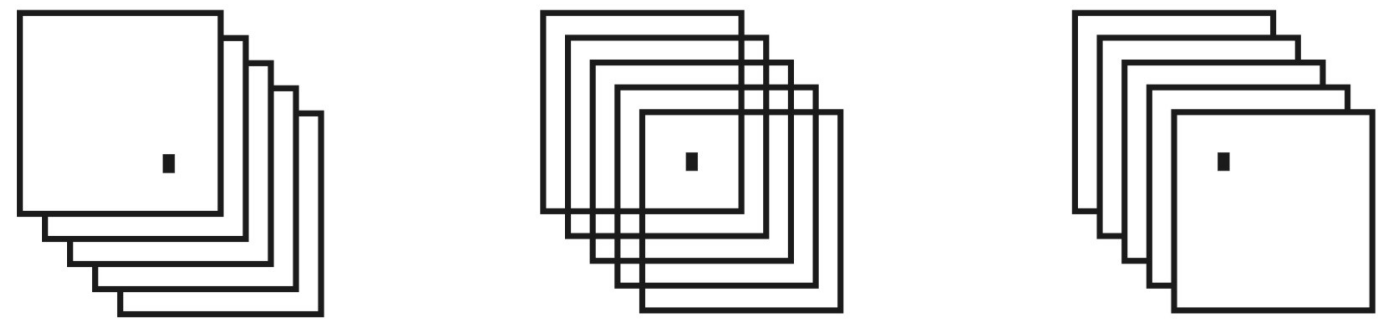

Figure 6. Example of possible perceptual modulators of bistable perception.

The bistable image (in the middle) is called The overlapping squares. Image retrieved January $30^{t h}$, 2018 from: http:// psycnet.apa.org/ftasset/journals/bul/130/5/images/bul_130_5_748_fig5a.gif.Thebistable

perceptual reversals that arise during bistable perception (Kanai, Carmel, Bahrami, \& Rees, 2011). It has also been found that the intraparietal sulcus plays a very important role in perceptual stabilization during bistable perception, while the dorsolateral prefrontal cortex is involved in the appearance of perceptual alternation (Vernet, Brem, Farzan, \& Pascual-Leone, 2015). The upper parietal lobe works as a regulator between alternation and stabilization; it is an area of the brain that operates as an integrator of neural activity during bistability and binocular rivalry, at least in perspective bistable images like Necker's cube (Baker, Karapanagiotidis, Coggan, Wailes-Newson, \& Smallwood, 2015). On the other hand, several recent studies have stated that there are correlations between perceptual alternations and the volume of gray matter in areas of the upper parietal lobe and with the concentration of gamma-aminobutyric acid (GABA) in the occipital lobe (Sandberg et al., 2016).

Perceptual reversals are related to certain activations in the extraestriate visual area; likewise, there are certain cortical areas that are involved in perceptual alternations, particularly the lower frontal cortex and the lower pari- etal cortex (Sterzer \& Rees, 2009). Nevertheless, apart from being associated with perceptual transitions during the observation of bistable images, the activity in the frontal cortex and in the parietal cortex is also involved in the stabilization of the percept (Sterzer et al., 2009). Specific regions of the occipital lobe and the parietal lobe show an important activation, raising the question of whether the frontal areas are the ones that exclusively initiate perceptual alternation (Castelo-Branco \& Castelhano, 2015). It has been stated that frontoparietal areas play a role in perceptual alternations. Additionally, it has been suggested that the right frontal and parietal cortex play a causal role in perceptual reversals (Weilnhammer, Ludwig, Hesselmann, \& Sterzer, 2013). Brain imaging studies show that a broad network of primarily righthemisphere frontal and parietal areas is activated around the time of perceptual reversals (Kleinschmidt et al., 1998). As can be seen, there is a connection between all these areas and the alternations during bistable perception, although their role as a causative factor has been a matter of debate (Weilnhammer et al., 2013).

With regard to the neural activity that is observed 
during such alternations, it has been stated that there are diverse areas that participate depending on the type of reversible images observed (Sterzer et al., 2009). Thus, for bistable images of a perspective type (like the Necker's cube), or for bistable images that admit perceptual and diverse percepts in respect to the perception of orientation and movement (like the Nobuyoki Kayahara ballerina), neural activity is present in the areas of the frontal and parietal lobes, in visual areas related to movement and also in areas of the extraestriate visual cortex. For perceptual reversals referred to bistable images of semantic content, an important activity is registered in the areas of the ventral stream (via occipital lobe to temporal lobe), areas that are associated with the recognition of shapes in terms of identifying what they are (Sterzer et al., 2009). That kind of neural activity leads to top-down processes in the recognition of percepts in terms of its semantic content. It has also been found that the balance between staying in a percept and jumping from one to another causes a dynamic interaction between the right intraparietal anterior sulcus and the prefrontal dorsolateral cortex (Vernet et al., 2015). Furthermore, reports refer to the correlations between the perceptual dynamics manifested during bistable perception in areas of the upper parietal lobe (Megumi et al., 2015; Sandberg et al., 2016). Moreover, it has been found that injuries in the lateral geniculate nucleus (thalamus) have a detrimental effect on experiencing perceptual reversibilities (Munar, Rosselló, Maiche, Travieso, \& Nadal, 2008). Likewise, damages to the frontal lobe, particularly right frontal injuries, reduce the transition of perceptions while observing reversible figures (Meenan \& Miller, 1994; Ricci \& Blundo, 1990).

\subsection{Perceptual reversals and binocular rivalry}

Several studies have indicated that the processes that are involved in the emergence of perceptual reversals are linked to binocular rivalry. Basically, binocular rivalry consists in the alternate perception between the different images that are exposed to each eye (Brascamp, Klink, \& Levelt, 2015; Carroll \& Bressloff, 2014; Mishra \& Hillyard, 2009; Mudrik, Deouell, \& Lamy, 2011; Takase, Yukumatsu, \& Bingushi, 2013). Thus, when one image is exposed to one eye and a different one is exposed to the other eye, instead of the images superimposing, one of them is seen for a few moments followed by the other. Thus, perception alternates between those two images at irregular intervals. This is typical of the perception that can be appreciated in the observation of bistable images, where the phenomenon of perceptive dominance arises. In addition, it has been shown that binocular rivalry plays an important role in perceptual reversibility, and its operativity impacts the perceptual reversals (van Dam \& van Ee, 2006; Weilnhammer et al., 2014). Moreover, there is a certain interaction between eye movements and perceptual alternations, so that such alternations can sometimes be manifested without there necessarily being eye movements, based on the fact that there is interdependence between the oculomotor activity and the voluntary control of perceptual reversals (Gale \& Findlay, 1983). Blinking can even influence perceptual transitions, given the difference manifested between the proximal stimulus presented before and after each blink (Baker \& Graf, 2010). On the other hand, binocular rivalry has been assumed as a low-level process (referring to interocular competition and the stimulus capacity), and also as a high-level process when it is linked to proceedings where an interocular grouping has emerged (de Weert, Snoeren, \& Koning, 2005). Functional brain-imaging studies of house-face binocular rivalry and Rubin's vase-face illusion have repeatedly reported face perception-dependent activity in the right fusiform gyrus. It has been also stated that high-level cortical structures play a role in perceptual rivalry (Ngo, Liu, Tilley, Pettigrew, \& Miller, 2008). Despite the fact that interocular conflicts that encourage binocular rivalry often occur, the perceptions are stable and seldom switch between dissimilar images. This fact can indicate that visual processing might inhibit perceptual rivalry (Takase et al., 2013). On the other hand, binocular rivalry is linked to attention modulation due to perceptual alternations during the competing process that are affected by the surrounding stimulus (Fukuda \& Blake, 1992). The contrast of the rival target is a factor by which the dominance of a monocular image accesses awareness (Castelo-Branco \& Castelhano, 2015). Competitive stimulus selections through visual attention are processed in the visual cortex with a selectivity that can be observed in higher cortical areas like V4, MT and IT (Mishra \& Hillyard, 2009). Endogenous attention extends the duration of dominance during rivalry when it is deployed to specific features of the competitive stimulus. Likewise, the selection of a rivalrous grating to be dominant in terms of perception can be made by both involuntary and voluntary attention (Hancock \& Andrews, 2007). As Mishra and Hillyard (2009) said, competition during binocular rivalry could be "an example of a more general attentional mechanism within the visual system".

\subsection{Perceptual bistability associated with the theoret- ical model of high and low levels}

It must be estimated that perceptual reversals can be explained through the theoretical model of high and low levels. As stated Sterzer and Rees (2009), there is a correspondence between high and low levels theory and bottom-up and top-down modulation of perception, respectively. As can be seen in Figure 5, there are two ways in which perceptual reversibility appears: option A, associated with the explanatory theory of low level, which suggests that spontaneous alternations take place in the visual cortex. This option is the foundation of bottom-up processing. The bottom-up explanatory theory of perceptual bistability states that a perceptual reversal 
occurs through mediation of an adaptation of the sensorial mechanisms; the activity of a perceptual mechanism sustains a specific perceptual configuration until, due to fatigue, the competitor percept emerges sustained in another mechanism (Intaitè et al., 2010; Kogo et al., 2015).

As far as option B is concerned (high level), in order to have perceptual reversibility-that is to say, the appearance of perceptual reversals-there is a dependence on central processing that can involve brain regions in the frontal area and elsewhere Sterzer and Rees (2009). The top-down processes emerge from this last model, which are related to psychological aspects of the individual (Barrera \& Calderón, 2013). What is more, it has been found that perceptual reversibility is connected to this type of proceeding (Long \& Toppino, 2004).

Evidence of the impact of top-down and bottom-up processes on bistable perception has been also reported when an image that does not accept perceptual reversibility is presented to the subjects before the ambiguous figure has been shown so that the knowledge obtained with the revision of the first contributes to the perception of the alternative percept, which suggests an adaptation effect (Kornmeier \& Bach, 2005; Qiu et al., 2009; Rock, Hall, \& Davis, 1994). In brief, it has been shown that bistable perception implies both types of processing (Kornmeier \& Bach, 2006).

\subsection{Bottom-up modulation and semantic modulation}

Gale and Findlay (1983) demonstrated that there are critical areas within a bistable image that favor perception of each of the possible percepts. After a thorough analysis of the critical lines that constitute the bistable image My girlfriend or my mother-in-law, they suggested that certain traits of the image enable the observer to perceive one image more easily than the other (young woman or old woman). After a graphic synthesis of the original Boring image, their study pointed out four specific areas, each one of them with visual and useful information for the recognition of either precept. On the basis of this study it was inferred that specific areas of attention favor one percept over the other (Hsiao et al., 2012). In other words, specific areas and certain aspects that constitute a bistable image facilitate the perception of a precept, and when an observer fixes their gaze in such areas and characteristics, the recognition of the percept emerges (Gale \& Findlay, 1983; García-Pérez, 1989). This type of perceptual modulation is known as the bottom-up factor, because the characteristic (or the modulator factor) entails a sensorial processing without the need for information that can imply a semantic priming effect (Meng \& Tong, 2004).

The perception of certain bistable images can be modulated from a disambiguation, where the implementation of an effect of perceptual priming can be experienced. Disambiguation implies the previous exposition of each possible percept of the bistable image (with zero possibility of bistability). Such exposition contributes to the perception of the previous disambiguated percept at the time of the observation of the bistable image (Qiu et al., 2009). As can be seen in Figure 6, the outer images become disambiguators of the ambiguous image, exposed in the middle (Intaitè et al., 2013).

In addition to this, intermittency paradigms have also been used to study perceptual bistability. In essence, this paradigm proposes the intermittent exposition of bistable visual stimuli, where it is evident that the dominant percept tends to keep manifesting itself after intermittency (usually areas in white), although it is warned that the duration of the interruption is critical (Kogo et al., 2015). Furthermore, there is a sort of modulation that involves the phenomenon of semantic congruency: when an observer is looking at an In meaning-content reversals bistable image, their perception of one of the possible percepts of the image can be influenced if they are simultaneously listening to an audio that refers to that particular percept. This is a crossmodal stimulation model, which also leads to a crossmodal perception (Lalanne \& Lorenceau, 2004). The semantic congruency is marked by the relation in terms of the semantic content between the auditory stimulus and what the image depicts (Balcetis \& Dale, 2007; Feist \& Gentner, 2007; Goolkasian \& Woodberry, 2010; Hsiao et al., 2012; Smith, Grabowecky, \& Suzuki, 2007). As was mentioned before, this type of modulation entails a crossmodal semantic congruency, where the modulation phenomenon emerges due to the association between the information provided from different sensorial modalities. This is how visual information and auditory stimulation are assumed as a unity by the observer. This notion is known as unity assumption (Vatakis \& Spence, 2007).

Conversely, it is possible to find unimodal modulation models (perceptual and semantic), for which a previously disambiguated image is exposed before the bistable image is shown. In this scenario, the information provided in the first exposition impacts the later perception of the bistable figure (Qiu et al., 2009).

\subsection{Other studies conducted on bistable images (or based on bistable perception)}

The particular features that define bistable figures allow their use in psychological research in topics related to attention and visual perception, to questions related to some disorders. In this regard, bistable stimulation may be involved in the field of psychopathology, taking into account that some disorders are linked to deficits in attentional control. Aydin, Strang, and Manahilov (2013) indicated that the paradigm of perceptual rivalry is a useful tool for exploring deficits in attentional control. For instance, Krug, Brunskill, Scarna, Goodwin, and Parker (2008), found that patients with bipolar disorder made fewer perceptual reversals than people without psychi- 
atric diagnoses. Furthermore, some studies on perceptual organization in schizophrenia spectrum disorders have shown evidence for and against a perceptual organization deficit (Uhlhaas \& Silverstein, 2005). Models of cognition that have appeared recently have also advanced understandings of the underlying pathophysiological processes of perceptual organization dysfunction in schizophrenia spectrum disorders. To some extent, deficits in perceptual organization may be part of an important disturbance in the integration of information across space and time. In other words, the perception of reality is not constant. Regarding this, the use of bistable perception has the potential to be taken into account as an alternative in the treatment of schizophrenic patients. Basar-Eroglu, Mathes, Khalaidovski, Brand, and Schmiedt-Fehr (2016) showed that pathological changes of alpha activity during visual bistable perception in schizophrenia could provide a proper model to study cognitive and sensorial processes and the likely impact of perceptual failures owing to psychiatric illness. Schizophrenia patients have impairments at several levels of cognition such as visual attention, perception, and social cognition (Heinrichs \& Zakzanis, 1998; Matsumoto, Takahashi, Murai, \& Takahashi, 2015), whereas a healthy person is able to experience bistable perception by stabilizing the current percept or by initiating perceptual reversals. McBain, Norton, Kim, and Chen (2011) indicated that patients with schizophrenia show altered processing of bistable images.

The features of bistable stimuli have also allowed them to be implemented as a research instrument in diverse psychological phenomena. Particularly, studies on bilingualism associated with perceptual capacities have found a cognitive mechanism in perceptual bistability that implies an analogous flexibility in bilingual minds (Bialystok \& Shapero, 2005). Moreover, the evaluation of executive functions linked to perceptual processes resorts to perceptual bistability to determine the effect of the decrease of certain cognitive abilities, including the analysis of variables like age. It has been found, for example, that there are correlations between age and the capacity to make spontaneous perceptual alternations; when a person reaches a certain age, this ability diminishes (Aydin et al., 2013).

Another study aimed at testing the role that is played by interhemispheric competition through the corpus callosum while alternating the percepts of bistable images. Conducted by Fagard et al. (2008), this study showed that the rate of perceptual reversals can be mediated by the corpus callosum. According to the researchers, the role that this interhemispheric connection plays within bistable perception phenomena might be attentional, given the fact that the corpus callosum is involved in the regulation of attention processes where the two hemispheres are implicated. Based on the results, they suggested that interhemispheric interaction related to bistable perception is linked to both low levels and high levels of processing.

On the other hand, bistable images have also been used to establish the effect of the gravitational orientation of body on visual perception. In this regard, different studies have been aimed at establishing the effect caused by body position on perceptual processes during the observation of bistable images. The findings of Yamamoto and Yamamoto (2006) suggested that the perception of bistable images is modulated by vertical gravity, a fact that can imply a multimodal integration of vestibular, proprioceptive, and tactile inputs. The findings of Clément and Eckardt (2005) show that the perceptual reversibility of perspective bistable images takes longer when subjects are laying down than when they are positioned vertically. Another study observed how depth perception and the perceptual predominance of bistable figures are altered when the human body is in conditions of micro or hyper gravity. It was found that gravity has an effect on the stability of the dominant percepts (Clément \& Demel, 2012). Finally, bistable images have been used to study insightfulness and creativity, as people who are able to discover the different percepts that are included in an ambiguous image are also good at solving creative problems (Laukkonen \& Tangen, 2017). Selective attention and its relation to bilingualism have also been studied with bistable images tasks. In this regard, it has been found that bilinguals are better at perceiving ambiguous images than monolinguals, suggesting that selective attention is implicated in the way people complete tasks connected to alternations while thinking or perceiving (Chung-Fat-Yim, Sorge, \& Bialystok, 2017).

\section{Conclusions}

The use of bistable images has allowed them to become a suitable paradigmatic resource for the instrumentalization of experimental tasks within the scope of basic psychological processes. Aside from contributing in the modeling of studies referred to attention, sensation, perception and memory, bistable perception itself has a great usage possibility, not only in the context of cognitive science, but also in neuropsychology and neurosciences. The singularity of the phenomenon allows researchers to study a large number of psychological factors, transcending the frontier of what is merely perceptual. On the other hand, studies conducted on bistable images have implied the use of diverse techniques and instruments, most of them non-invasive. Several scientific findings have been useful in understanding bistable perception as a psychological phenomenon, from neurological foundations to the cognitive processes that are implicated while ambiguous images are being seen. Visual search tasks can use bistable images to study and understand multiple psychological processes. Given the fact that several 
psychological mechanisms are linked to the observation of bistable images and taking into account that multiple areas of the brain are activated while observing these images, it is imperative to carry on understanding the modus operandi of this phenomenon, in order to keep making progress in the discovery of the functioning of the human psyche.

\section{References}

Aydin, S., Strang, N. C., \& Manahilov, V. (2013). Agerelated deficits in attentional control of perceptual rivalry. Vision Research, 77, 32-40. doi: https:// doi.org/10.1016/j.visres.2012.11.010

Baker, D. H., \& Graf, E. W. (2010). Extrinsic factors in the perception of bistable motion stimuli. Vision Research, 50(13), 1257-1265. doi: https://doi.org/ 10.1016/j.visres.2010.04.016

Baker, D. H., Karapanagiotidis, T., Coggan, D. D., Wailes-Newson, K., \& Smallwood, J. (2015). Brain networks underlying bistable perception. NeuroImage, 119, 229-234. doi: https://doi.org/10.1016/ j.neuroimage.2015.06.053

Balcetis, E., \& Dale, R. (2007). Conceptual set as a top-down constraint on visual object identification. Perception, 36(4), 581-595. doi: https:// doi.org/10.1068/p5678

Barrera, M., \& Calderón, L. (2013). Notes for supporting an epistemological neuropsychology: contributions from three perspectives. International Journal of Psychological Research, 6(2), 107-118.

Basar-Eroglu, C., Mathes, B., Khalaidovski, K., Brand, A., \& Schmiedt-Fehr, C. (2016). Altered alpha brain oscillations during multistable perception in schizophrenia. International Journal of Psychophysiology, 103, 118-128. doi: https://doi.org/ 10.1016/j.ijpsycho.2015.02.002

Bialystok, E., \& Shapero, D. (2005). Ambiguous benefits: The effect of bilingualism on reversing ambiguous figures. Developmental Science, 8(6), 595-604. doi: https://doi.org/10.1111/j.1467-7687.2005.00451.x

Borisyuk, R., Chik, D., \& Kazanovich, Y. (2009). Visual perception of ambiguous figures: synchronization based neural models. Biological Cybernetics, 100(6), 491-504. doi: 10.1007/s00422-009-0301-1

Borisyuk, R., \& Hoppensteadt, F. (2004). A theory of epineuronal memory. Neural Networks, 17(10), 1427-1436. doi: https://doi.org/10.1016/j.neunet .2004 .07 .006

Brascamp, J. W., Klink, P. C., \& Levelt, W. J. M. (2015). The "laws" of binocular rivalry: 50 years of Levelt's propositions. Vision Research, 109, 20-37. doi: https://doi.org/10.1016/j.visres.2015.02.019

Brouwer, G. J., \& van Ee, R. (2006). Endogenous influences on perceptual bistability depend on exogenous stimulus characteristics. Vision Research,
46(20), 3393-3402. doi: https://doi.org/10.1016/ j.visres.2006.03.016

Carroll, S. R., \& Bressloff, P. C. (2014). Binocular rivalry waves in a directionally selective neural field model. Physica D: Nonlinear Phenomena, 285, 8-17. doi: https://doi.org/10.1016/j.physd.2014.07.002

Castelo-Branco, M., \& Castelhano, J. (2015). Perceptual decision making. In A. W. Toga (Ed.), Brain Mapping (p. 401 - 408). Waltham: Academic Press. doi: https://doi.org/10.1016/B978-0-12-397025-1 .00261-X

Chung-Fat-Yim, A., Sorge, G. B., \& Bialystok, E. (2017). The relationship between bilingualism and selective attention in young adults: evidence from an ambiguous figures task. The Quarterly Journal of Experimental Psychology, 70(3), 366-372. doi: https://doi.org/10.1080/17470218.2016.1221435

Clément, G., \& Demel, M. (2012). Perceptual reversal of bi-stable figures in microgravity and hypergravity during parabolic flight. Neuroscience Letters, 507(2), 143-146. doi: https://doi.org/10.1016/ j.neulet.2011.12.006

Clément, G., \& Eckardt, J. (2005). Influence of the gravitational vertical on geometric visual illusions. Acta Astronautica, 56(9-12), 911-917. doi: https:// doi.org/10.1016/j.actaastro.2005.01.017

Cumming, B. G., \& Parker, A. J. (1997). Responses of primary visual cortical neurons to binocular disparity without depth perception. Nature, 389(6648), 280. doi: $10.1038 / 38487$

Denham, S., Bendixen, A., Mill, R., Tóth, D., Wennekers, T., Coath, M., ... Winkler, I. (2012). Characterising switching behaviour in perceptual multi-stability. Journal of Neuroscience Methods, 210(1), 79 - 92. doi: https://doi.org/10.1016/j.jneumeth.2012.04 .004

de Weert, C. M. M., Snoeren, P. R., \& Koning, A. (2005). Interactions between binocular rivalry and Gestalt formation. Vision Research, 45(19), 2571-2579. doi: https://doi.org/10.1016/j.visres.2005.04.005

Fagard, J., Sacco, S., Yvenou, C., Domellöf, E., Kieffer, V., Tordjman, S., ... Mamassian, P. (2008). The role of the corpus callosum in the perception of reversible figures in children. Vision $R e$ search, 48(23-24), 2451-2455. doi: https://doi.org/ $10.1016 /$ j.visres.2008.08.007

Feist, M. I., \& Gentner, D. (2007). Spatial language influences memory for spatial scenes. Memory \& Cognition, 35(2), 283-296. doi: 10.3758/BF03193449

Fukuda, H., \& Blake, R. (1992). Spatial interactions in binocular rivalry. Journal of Experimental Psychology: Human Perception and Performance, 18(2), 362. doi: http://dx.doi.org/10.1037/ 0096-1523.18.2.362

Gale, A. G., \& Findlay, J. M. (1983). Eye movement 
patterns in viewing ambiguous figures. Eye movements and psychological functions: International views, $145-168$.

García-Pérez, M. A. (1989). Visual inhomogeneity and eye movements in multistable perception. Attention, Perception, \& Psychophysics, 46(4), 397-400. doi: https://link.springer.com/content/ pdf/10.3758/BF03204995.pdf

García-Pérez, M. A. (1992). The role of eye movements in perceptual processes. In E. Chekaluk \& K. Llewellyn (Eds.), (Vol. 88, pp. 73-109). Amsterdam - ondon - New York - Tokyo: NorthHolland: Elsevier. doi: https://doi.org/10.1016/ S0166-4115(08)61743-4

Goolkasian, P., \& Woodberry, C. (2010). Priming effects with ambiguous figures. Attention, Perception, 6) Psychophysics, 72(1), 168-178. doi: https:// link.springer.com/article/10.3758/APP.72.1.168

Gori, S., Giora, E., \& Pedersini, R. (2008). Perceptual multistability in figure-ground segregation using motion stimuli. Acta Psychologica, 129(3), 399-409. doi: https://doi.org/10.1016/j.actpsy.2008.09.004

Grossmann, J. K., \& Dobbins, A. C. (2006). Competition in bistable vision is attribute-specific. Vision Research, 46(3), 285-292. doi: https://doi.org/ $10.1016 /$ j.visres.2005.06.002

Hancock, S., \& Andrews, T. J. (2007). The role of voluntary and involuntary attention in selecting perceptual dominance during binocular rivalry. Perception, 36(2), 288-298. doi: https://doi.org/10.1068/ p5494

Heinrichs, R. W., \& Zakzanis, K. K. (1998). Neurocognitive deficit in schizophrenia: a quantitative review of the evidence. Neuropsychology, 12(3), 426. doi: 10.1037/0894-4105.12.3.426

Hsiao, J.-Y., Chen, Y.-C., Spence, C., \& Yeh, S.-L. (2012). Assessing the effects of audiovisual semantic congruency on the perception of a bistable figure. Consciousness and Cognition, 21(2), 775-787. doi: https://doi.org/10.1016/j.concog.2012.02.001

Intaitè, M., Koivisto, M., \& Castelo-Branco, M. (2014). Event-related potential responses to perceptual reversals are modulated by working memory load. Neuropsychologia, 56, 428-438. doi: https:// doi.org/10.1016/j.neuropsychologia.2014.02.016

Intaitè, M., Koivisto, M., Rukšènas, O., \& Revonsuo, A. (2010). Reversal negativity and bistable stimuli: Attention, awareness, or something else? Brain and Cognition, $74(1), 24-34$. doi: https://doi.org/ 10.1016/j.bandc.2010.06.002

Intaitè, M., Noreika, V., Šoliūnas, A., \& Falter, C. M. (2013). Interaction of bottom-up and top-down processes in the perception of ambiguous figures. Vision Research, 89, 24-31. doi: https://doi.org/ $10.1016 /$ j.visres.2013.06.011
Jackson, S., Cummins, F., \& Brady, N. (2008). Rapid perceptual switching of a reversible biological figure. PloS one, 3(12), 1-15. doi: https://doi.org/10 .1371 /journal.pone.0003982

Kanai, R., Carmel, D., Bahrami, B., \& Rees, G. (2011). Structural and functional fractionation of right superior parietal cortex in bistable perception. Current Biology, 21(3), R106-R107. doi: https:// doi.org/10.1016/j.cub.2010.12.009

Kleinschmidt, A., Büchel, C., Zeki, S., \& Frackowiak, R. S. J. (1998). Human brain activity during spontaneously reversing perception of ambiguous figures. Proceedings of the Royal Society of London B: Biological Sciences, 265(1413), 2427-2433. doi: 10.1098/rspb.1998.0594

Kogo, N., Hermans, L., Stuer, D., van Ee, R., \& Wagemans, J. (2015). Temporal dynamics of different cases of bi-stable figure-ground perception. Vision Research, 106, 7-19. doi: https://doi.org/10.1016/ j.visres.2014.10.029

Kornmeier, J., \& Bach, M. (2005). The Necker cube-an ambiguous figure disambiguated in early visual processing. Vision Research, 45(8), 955-960. doi: https://doi.org/10.1016/j.visres.2004.10.006

Kornmeier, J., \& Bach, M. (2006). Bistable perception - along the processing chain from ambiguous visual input to a stable percept. International Journal of Psychophysiology, 62(2), 345-349. doi: https://doi.org/10.1016/j.ijpsycho.2006.04.007

Kornmeier, J., Hein, C. M., \& Bach, M. (2009). Multistable perception: when bottom-up and top-down coincide. Brain and Cognition, 69(1), 138-147. doi: https://doi.org/10.1016/j.bandc.2008.06.005

Krug, K., Brunskill, E., Scarna, A., Goodwin, G. M., \& Parker, A. J. (2008). Perceptual switch rates with ambiguous structure-from-motion figures in bipolar disorder. Proceedings of the Royal Society of London B: Biological Sciences, 275(1645), 1839-1848. doi: $10.1098 /$ rspb.2008.0043

Lalanne, C., \& Lorenceau, J. (2004). Crossmodal integration for perception and action. Journal of Physiology-Paris, 98(1-3), 265-279. doi: https:// doi.org/10.1016/j.jphysparis.2004.06.001

Laukkonen, R. E., \& Tangen, J. M. (2017). Can observing a Necker cube make you more insightful? Consciousness and Cognition, 48, 198-211. doi: https://doi.org/10.1016/j.concog.2016.11.011

Leopold, D. A., \& Logothetis, N. K. (1999). Multistable phenomena: changing views in perception. Trends in cognitive sciences, 3(7), 254-264. doi: https:// doi.org/10.1016/S1364-6613(99)01332-7

Liu, C.-H., Tzeng, O. J. L., Hung, D. L., Tseng, P., \& Juan, C.-H. (2012). Investigation of bistable perception with the "silhouette spinner": Sit still, spin the dancer with your will. Vision Research, 
60, 34-39. doi: https://doi.org/10.1016/j.visres .2012 .03 .005

Long, G. M., \& Batterman, J. M. (2012). Dissecting perceptual processes with a new tri-stable reversible figure. Perception, 41(10), 1163-1185. doi: https:// doi.org/10.1068/p7313

Long, G. M., \& Toppino, T. C. (1981). Multiple representations of the same reversible figure: Implications for cognitive decisional interpretations. Perception, 10(2), 231-234. doi: https://doi.org/10.1068/ p100231

Long, G. M., \& Toppino, T. C. (2004). Enduring interest in perceptual ambiguity: alternating views of reversible figures. Psychological bulletin, 130(5), 748. doi: 10.1037/0033-2909.130.5.748

Matsumoto, Y., Takahashi, H., Murai, T., \& Takahashi, H. (2015). Visual processing and social cognition in schizophrenia: relationships among eye movements, biological motion perception, and empathy. Neuroscience research, 90, 95-100. doi: https://doi.org/10.1016/j.neures.2014.10.011

McBain, R., Norton, D. J., Kim, J., \& Chen, Y. (2011). Reduced cognitive control of a visually bistable image in schizophrenia. Journal of the International Neuropsychological Society, 17(3), 551-556. doi: https://doi.org/10.1016/j.ijpsycho.2015.02.002

Meenan, J. P., \& Miller, L. A. (1994). Perceptual flexibility after frontal or temporal lobectomy. Neuropsychologia, 32(9), 1145-1149. doi: https://doi.org/ 10.1016/0028-3932(94)90159-7

Megumi, F., Bahrami, B., Kanai, R., \& Rees, G. (2015). Brain activity dynamics in human parietal regions during spontaneous switches in bistable perception. NeuroImage, 107, 190-197. doi: https://doi.org/ 10.1016/j.neuroimage.2014.12.018

Meng, M., \& Tong, F. (2004). Can attention selectively bias bistable perception? Differences between binocular rivalry and ambiguous figures. Journal of Vision, 4(7), 2-2. doi: 10.1167/4.7.2

Meso, A. I., \& Masson, G. S. (2015). Dynamic resolution of ambiguity during tri-stable motion perception. Vision research, 10\%, 113-123. doi: https://doi.org/ 10.1016/j.visres.2014.12.015

Mishra, J., \& Hillyard, S. A. (2009). Endogenous attention selection during binocular rivalry at early stages of visual processing. Vision research, 49(10), 1073-1080. doi: https://doi.org/10.1016/j.visres .2008 .02 .018

Moreno-Bote, R., Rinzel, J., \& Rubin, N. (2007). Noiseinduced alternations in an attractor network model of perceptual bistability. Journal of neurophysiology, 98(3), 1125-1139. doi: https://doi.org/ $10.1152 /$ jn.00116.2007

Mudrik, L., Deouell, L. Y., \& Lamy, D. (2011). Scene congruency biases binocular rivalry. Consciousness and cognition, 20(3), 756-767. doi: https://doi.org/ 10.1016/j.concog.2011.01.001

Munar, E., Rosselló, J., Maiche, A., Travieso, D., \& Nadal, M. (2008). Manual de neuropsicología. In V. Editores (Ed.), (pp. 59-96). Barcelona. doi: https://dialnet.unirioja.es/servlet/articulo ?codigo $=3423906$

Munhall, K. G., Ten Hove, M. W., Brammer, M., \& Paré, M. (2009). Audiovisual integration of speech in a bistable illusion. Current Biology, 19(9), 735-739. doi: 10.1016/j.cub.2009.03.019

Naber, M., Gruenhage, G., \& Einhäuser, W. (2010). Tristable stimuli reveal interactions among subsequent percepts: Rivalry is biased by perceptual history. Vision Research, 50(8), 818-828. doi: https:// doi.org/10.1016/j.visres.2010.02.004

Ngo, T. T., Liu, G. B., Tilley, A. J., Pettigrew, J. D., \& Miller, S. M. (2008). The changing face of perceptual rivalry. Brain Research Bulletin, 75(5), 610-618. doi: https://doi.org/10.1016/ j.brainresbull.2007.10.006

Okazaki, M., Kaneko, Y., Yumoto, M., \& Arima, K. (2008). Perceptual change in response to a bistable picture increases neuromagnetic beta-band activities. Neuroscience Research, 61(3), 319-328. doi: 10.1016/j.neures.2008.03.010

Piantoni, G., Romeijn, N., Gomez-Herrero, G., Werf, Y. D., \& Someren, E. J. W. (2017). Alpha power predicts persistence of bistable perception. Scientific Reports, 7(1), 5208. doi: 10.1038/ s41598-017-05610-8

Pressnitzer, D., \& Hupé, J.-M. (2006). Temporal dynamics of auditory and visual bistability reveal common principles of perceptual organization. Current biology, 16(13), 1351-1357. doi: https://doi.org/10.1016/j.cub.2006.05.054

Qiu, J., Wei, D., Li, H., Yu, C., Wang, T., \& Zhang, Q. (2009). The vase-face illusion seen by the brain: An event-related brain potentials study. International Journal of Psychophysiology, 74(1), 69-73. doi: https://doi.org/10.1016/j.ijpsycho.2009.07.006

Ricci, C., \& Blundo, C. (1990). Perception of ambiguous figures after focal brain lesions. Neuropsychologia, 28(11), 1163-1173. doi: https://doi.org/10.1016/ 0028-3932(90)90052-P

Rock, I., Hall, S., \& Davis, J. (1994). Why do ambiguous figures reverse? Acta Psychologica, 87(1), 33-59. doi: https://www.ncbi.nlm.nih.gov/ pubmed/7985524

Sandberg, K., Barnes, G. R., Bahrami, B., Kanai, R., Overgaard, M., \& Rees, G. (2014). Distinct MEG correlates of conscious experience, perceptual reversals and stabilization during binocular rivalry. Neuroimage, 100, 161-175. doi: https://doi.org/ $10.1016 /$ j.neuroimage.2014.06.023 
Sandberg, K., Blicher, J. U., Del Pin, S. H., Andersen, L. M., Rees, G., \& Kanai, R. (2016). Improved estimates for the role of grey matter volume and GABA in bistable perception. Cortex, 83, 292-305. doi: https://doi.org/10.1016/j.cortex.2016.08.006

Schauer, G., Kanai, R., \& Brascamp, J. W. (2016). Parietal theta burst TMS: Functional fractionation observed during bistable perception not evident in attention tasks. Consciousness and cognition, 40, 105-115. doi: https://doi.org/10.1016/j.concog .2016 .01 .002

Smith, E. L., Grabowecky, M., \& Suzuki, S. (2007). Auditory-visual crossmodal integration in perception of face gender. Current Biology, 17(19), 1680-1685. doi: https://doi.org/10.1016/j.cub .2007 .08 .043

Sterzer, P., Kleinschmidt, A., \& Rees, G. (2009). The neural bases of multistable perception. Trends in Cognitive Sciences, 13(7), 310-318. doi: 10.1016/ j.tics.2009.04.006

Sterzer, P., \& Rees, G. (2009). Bistable perception and consciousness. Encyclopedia of Consciousness, 93-106. doi: https://doi.org/10.1016/B978 -012373873-8.00011-6

Sterzer, P., Russ, M. O., Preibisch, C., \& Kleinschmidt, A. (2002). Neural correlates of spontaneous direction reversals in ambiguous apparent visual motion. Neuroimage, 15(4), 908-916. doi: https://doi.org/ 10.1006/nimg.2001.1030

Takase, S., Yukumatsu, S., \& Bingushi, K. (2013). Perceptual dominance during binocular rivalry is prolonged by a dynamic surround. Vision research, 92, 33-38. doi: https://doi.org/10.1016/ j.visres.2013.09.002

Uhlhaas, P. J., \& Silverstein, S. M. (2005). Perceptual organization in schizophrenia spectrum disorders: empirical research and theoretical implications. Psychological Bulletin, 131(4), 618. doi: 10.1037/0033-2909.131.4.618

van Dam, L. C. J., \& van Ee, R. (2006). The role of saccades in exerting voluntary control in perceptual and binocular rivalry. Vision research, 46(6-7), 787-799. doi: https://doi.org/10.1016/j.visres.2005 .10 .011

van Loon, A. M., Knapen, T., Scholte, H. S., JohnSaaltink, E. S., Donner, T. H., \& Lamme, V. A. F. (2013). GABA shapes the dynamics of bistable perception. Current Biology, 23(9), 823-827. doi: https://doi.org/10.1016/j.cub.2013.03.067

Vatakis, A., \& Spence, C. (2007). Crossmodal binding: Evaluating the "unity assumption" using audiovisual speech stimuli. Perception \& Psychophysics, 69(5), 744-756. doi: https://doi.org/10.3758/ BF03193776

Vernet, M., Brem, A.-K., Farzan, F., \& Pascual-Leone, A.
(2015). Synchronous and opposite roles of the parietal and prefrontal cortices in bistable perception: a double-coil TMS-EEG study. Cortex, 64, 78-88. doi: https://doi.org/10.1016/j.cortex.2014.09.021

Weilnhammer, V., Ludwig, K., Hesselmann, G., \& Sterzer, P. (2013). Frontoparietal cortex mediates perceptual transitions in bistable perception. Journal of Neuroscience, 33(40), 16009-16015. doi: https://doi.org/10.1523/JNEUROSCI.1418 $-13.2013$

Weilnhammer, V., Ludwig, K., Sterzer, P., \& Hesselmann, G. (2014). Revisiting the Lissajous figure as a tool to study bistable perception. Vision $R e$ search, 98, 107-112. doi: https://doi.org/10.1016/ j.visres.2014.03.013

Weilnhammer, V., Stuke, H., Hesselmann, G., Sterzer, P., \& Schmack, K. (2017). A predictive coding account of bistable perception-a model-based fMRI study. PLoS Computational Biology, 13(5), e1005536. doi: https://doi.org/10.1371/journal.pcbi.1005536

Xiaogang, W., Na, S., Lei, H., Yong, Z., Taiyong, B., \& Jiang, Q. (2017). Category selectivity of human visual cortex in perception of rubin face-vase illusion. Frontiers in Psychology. doi: https://doi.org/10.3389/fpsyg.2017.01543

Yamamoto, S., \& Yamamoto, M. (2006). Effects of the gravitational vertical on the visual perception of reversible figures. Neuroscience Research, 55(2), 218-221. doi: https://doi.org/10.1016/ j.neures.2006.02.014

Yeh, S.-L., Hsiao, J.-Y., Chen, Y.-C., \& Spence, C. (2011). Interplay of multisensory processing, attention, and consciousness as revealed by bistable figures. $i$-Perception, 2(8), 910-910. doi: https:// doi.org/10.1068/ic910 